\title{
Communication \\ Combination of the Glutaminyl Cyclase Inhibitor PQ912 (Varoglutamstat) and the Murine Monoclonal Antibody PBD-C06 (m6) Shows Additive Effects on Brain A $\beta$ Pathology in Transgenic Mice
}

\author{
Torsten Hoffmann ${ }^{1, *}$, Jens-Ulrich Rahfeld ${ }^{2}{ }^{(}$, Mathias Schenk ${ }^{2}{ }^{(}$, Falk Ponath ${ }^{3}$, Koki Makioka ${ }^{3}$, \\ Birgit Hutter-Paier ${ }^{4}$, Inge Lues ${ }^{1}$, Cynthia A. Lemere ${ }^{3}$ and Stephan Schilling ${ }^{2,5, *}$ \\ 1 Vivoryon Therapeutics N.V., Weinbergweg 22, 06120 Halle, Germany; ilues@me.com \\ 2 Fraunhofer Institute for Cell Therapy and Immunology, Department of Drug Design and Target Validation, \\ Weinbergweg 22, 06120 Halle, Germany; Jens-Ulrich.Rahfeld@izi.fraunhofer.de (J.-U.R.); \\ mathias.schenk@izi.fraunhofer.de (M.S.) \\ 3 Department of Neurology, Brigham and Women's Hospital, Harvard Medical School, 60 Fenwood Rd., \\ Boston, MA 02115, USA; falk.ponath@helmholtz-hiri.de (F.P.); makiokakoki@yahoo.co.jp (K.M.); \\ clemere@bwh.harvard.edu (C.A.L.) \\ 4 QPS Austria GmbH, Department of Neuropharmacology, Parkring 12, A-8074 Grambach, Austria; \\ Birgit.Hutter-Paier@qps.com \\ check for \\ updates \\ 5 Anhalt University of Applied Sciences, Bernburger Straße 55, 06366 Köthen, Germany \\ * Correspondence: Torsten.Hoffmann@vivoryon.com (T.H.); Stephan.schilling@izi.fraunhofer.de (S.S.)
}

Citation: Hoffmann, T.; Rahfeld J.-U.; Schenk, M.; Ponath, F.; Makioka, K.; Hutter-Paier, B.; Lues, I.; Lemere, C.A.; Schilling, S. Combination of the Glutaminyl Cyclase Inhibitor PQ912 (Varoglutamstat) and the Murine Monoclonal Antibody PBD-C06 (m6) Shows Additive Effects on Brain A $\beta$ Pathology in Transgenic Mice. Int. J. Mol. Sci. 2021, 22, 11791. https:// doi.org/10.3390/ijms222111791

Academic Editors: Botond Penke and Maria Laura Giuffrida

Received: 6 September 2021

Accepted: 27 October 2021

Published: 30 October 2021

Publisher's Note: MDPI stays neutral with regard to jurisdictional claims in published maps and institutional affiliations.

Copyright: () 2021 by the authors. Licensee MDPI, Basel, Switzerland. This article is an open access article distributed under the terms and conditions of the Creative Commons Attribution (CC BY) license (https:// creativecommons.org/licenses/by/ $4.0 /)$.
Abstract: Compelling evidence suggests that pyroglutamate-modified $A \beta$ (pGlu3-A $\beta ; A \beta N 3 p G$ ) peptides play a pivotal role in the development and progression of Alzheimer's disease (AD). Approaches targeting pGlu3-A $\beta$ by glutaminyl cyclase (QC) inhibition (Varoglutamstat) or monoclonal antibodies (Donanemab) are currently in clinical development. Here, we aimed at an assessment of combination therapy of Varoglutamstat (PQ912) and a pGlu3-A $\beta$-specific antibody (m6) in transgenic mice. Whereas the single treatments at subtherapeutic doses show moderate (16-41\%) but statistically insignificant reduction of $A \beta 42$ and pGlu-A $\beta 42$ in mice brain, the combination of both treatments resulted in significant reductions of $\mathrm{A} \beta$ by $45-65 \%$. Evaluation of these data using the Bliss independence model revealed a combination index of $\approx 1$, which is indicative for an additive effect of the compounds. The data are interpreted in terms of different pathways, in which the two drugs act. While PQ912 prevents the formation of pGlu3-A $\beta$ in different compartments, the antibody is able to clear existing pGlu3-A $\beta$ deposits. The results suggest that combination of the small molecule Varoglutamstat and a pE3A $\beta$-directed monoclonal antibody may allow a reduction of the individual compound doses while maintaining the therapeutic effect.

Keywords: glutaminyl cyclase inhibitor; anti-pyroglutamyl $\beta$-amyloid antibody; drug combination; Alzheimer's disease; hAPPsl $\times$ hQC mice; immunotherapy

\section{Introduction}

Dementia currently affects about 50 million people worldwide, representing the 5th leading cause of death [1]. Among the different causes of dementia, Alzheimer's disease (AD) is the most prominent. At the molecular level $\mathrm{AD}$ is characterized by two main pathological hallmarks in the brain, extracellular amyloid plaques, mainly consisting of different molecular forms of the APP derived amyloid- $\beta$ peptides, and formation of neurofibrillary tangles, composed of aggregated and hyperphosphorylated tau. According to the widely accepted amyloid hypothesis, formation of toxic $\mathrm{A} \beta$ oligomers and plaques precedes the tau pathology and is probably an early driver of the disease.

There are a couple of $A \beta$-directed monoclonal antibodies in the development of treatment of AD. Moreover, the recent promising results from clinical studies with e.g., 
Lecanemab (BAN2401), which targets large soluble A $\beta$ protofibrils, or Donanemab, which is directed to pGlu3-A $\beta$, and the accelerated approval of Aducanumab (a human monoclonal antibody preferentially binds $\mathrm{A} \beta$ oligomers and fibrils) provide prominent support for the amyloid hypothesis and give rise to the assumption that targeting $A \beta$ is a valid approach to developing treatments for Alzheimer's disease [2-5]. Among these antibody molecules, Donanemab recently showed significant removal of amyloid load and cognitive stabilization in a Phase 2 clinical trial [6,7]. Interestingly, this is also the first antibody in clinical development, which specifically binds pyroglutamate-modified amyloid peptides ( $p$ Glu3-A $\beta, A \beta N 3 p G$ ). In principle, the rationale to target $p G l u-A \beta$ is based on the observation of a particular toxicity of this molecular species of $A \beta$ [8-11]. Numerous studies have shown that formation of certain $N$-truncated $A \beta$ is followed by glutaminyl cyclase $(Q C)$ catalyzed conversion of glutamic acid into pyroglutamic acid to form pGlu-A $\beta$ [12-18]. This N-terminal modification induces changes in the molecular properties, likely driven by an increase of hydrophobicity, which, in turn, leads to accelerated formation of highly neurotoxic oligomers $[8,19]$. The toxic effect is potentially caused by a unique interaction of pGlu- $A \beta$ with receptors [20]. In addition, the pGlu-modified $A \beta$ is stabilized against $\mathrm{N}$-terminal degradation causing accumulation in AD brains [21]. Importantly, evidence was provided that the appearance of pGlu-A $\beta$ is interconnected with early stages of $A D$ development and its accumulation parallels cognitive decline [22,23].

Hence, approaches to target $\mathrm{pGlu}-\mathrm{A} \beta$ were suggested to interfere with $\mathrm{AD}$ progression. In principle, two strategies were discovered and successfully evaluated; reduction of pGlu3$A \beta$ formation by inhibition of $Q C$, or the application of anti-pGlu3-A $\beta$ antibodies [24-28]. In this regard, QC shows several characteristics that link its activity to AD development and progression. Under physiological conditions, the enzyme shows its highest expression in the brain $[29,30]$. Its expression has been shown to be significantly increased in AD, especially within the cortex [12,31]. The increase of activity parallels with the detection of pGlu-A $\beta[12,24]$. Accordingly, inhibition of $\mathrm{QC}$ has been shown to attenuate ADlike symptoms in mice dose-dependently [27] and Varoglutamstat, a first in-class QC inhibitor, has been proven safe and showed signs of efficacy in clinical phase 1 and 2 studies $[32,33]$. In contrast to the suppression of pGlu-formation by inhibition of $Q C$, the targeting with antibodies aims at clearance of $\mathrm{pGlu}-\mathrm{A} \beta$ after formation and/or the blocking of aggregation [34]. Hence, both treatment paradigms address the same goal by apparently different and independent molecular strategies. Therefore, we here aimed at a combination treatment to address, whether the effect of both compounds can be combined.

The result of our study in an AD mouse model overexpressing human amyloid precursor protein containing the Swedish and London mutation and human QC (hAPPsl $\times$ hQC) indicates an additive effect of both treatment strategies which might open up new avenues for an $\mathrm{AD}$ treatment by combining two disease-modifying $\mathrm{A} \beta$-directed therapies in a practical clinical setting in future.

\section{Results}

The aim of this study was to evaluate the combination effect of a glutaminyl cyclase inhibitor and a pGlu-specific antibody on the formation and clearance of pGlu-A $\beta$ in transgenic mice. Based on the results of previous studies [27], we selected a dose of $0.8 \mathrm{~g} / \mathrm{kg}$ PQ912 in chow, which translates in this study to a daily dose of $\approx 140 \mathrm{mg} / \mathrm{kg}$. The pGlu-specific antibody m6 (murine PBD-C06, IgG2a) was also investigated in detail in previous studies [34,35], however, these studies were not performed in the hAPPsl $\times \mathrm{hQC}$ transgenic mice, which harbor an increased pGlu-A $\beta$ formation. Hence, we first performed a dose-finding study with the $\mathrm{m} 6$ antibody to be able to select subtherapeutic doses of both treatments for the combination experiment.

\subsection{Dose Finding Study with m6 Antibody}

In this dose-finding study 150 and $500 \mu \mathrm{g}$ of the pGlu3-A $\beta$ specific $\mathrm{m} 6$ antibody were applied weekly i.p. for 16 weeks. The $\mathrm{m} 6$ treatment showed a dose-dependent significant 
decrease of A $\beta 42(p<0.05$ for both doses) and pGlu3-A $\beta 42(p<0.01$ for $500 \mu \mathrm{g}$ dose) levels in the soluble [Tris buffered solution (TBS)] brain fraction. Lower levels of both $A \beta$ forms were found in the insoluble [sodium dodecyl sulfate (SDS) + formic acid (FA)] brain fraction, too, but this decrease did not reach significance (Figure S1). From these results, we concluded that the lower $\mathrm{m} 6$ dose $(150 \mu \mathrm{g} /$ week) results in minimal efficacy (decrease in $A \beta)$, which may be improved with the combination with a $Q C$ inhibitor.

\subsection{Combination Treatment of $P Q 912$ and $m 6$ Antibody}

\subsubsection{Effect on Animal Health and Drug Levels}

In the combination study over 16 weeks all treatments including the combination were well tolerated. Body weight increase over treatment period indicated no statistically significant difference between groups (not shown). Brain levels of PQ912 and m6 antibody were determined at study end to check if these are comparable between the respective treatment groups (Figure S2). The mean brain concentrations of the $\mathrm{m} 6$ antibody were all in the same range and independent of the treatment or gender. Although, 2-way ANOVA indicates that gender influences the levels of PQ912 (lower levels in males, $p=0.004$ ) there are no differences in PQ912 level between the mono- and the combination therapy $(p=0.76)$.

\subsubsection{Development of A $\beta$ Pathology in 9- to 12.5-Month-Old APPslxhQC Mice}

Initially, we investigated the development of pathology (A $\beta$ deposition in brain) from baseline to the end of the 16-week treatment period and analyzed if the $A \beta$ levels in the isotype antibody treated control are comparable to that of the vehicle-treated control. Furthermore, sex was evaluated as a possible factor influencing pathology (Figure S3). Here, male mice showed much lower $\mathrm{A} \beta$ deposition compared with female animals. For females, a significant increase of $\mathrm{A} \beta 42(p \leq 0.002)$ and pGlu3-A $\beta 42(p<0.001)$ during the 16-week treatment period could be observed for the vehicle as well as for the isotype-treated group without a detectable difference between vehicle and isotype antibody treatment. In the male animals also a nominal increase of insoluble $\mathrm{A} \beta$ was observed in both groups compared to the baseline but this was less than half of that observed in females, and the differences to baseline were not significant. The low $p$-values of the interaction terms $(p=0.1$ and 0.03 for $\mathrm{A} \beta 42$ and pGlu3-A $\beta 42$, respectively) also indicate that the development of pathology was different between males and females in this experiment. Such a gender difference of $A \beta$ deposition rate was also described for other AD models e.g., APP23 mice [36]. Based on these results, we decided to use the isotype-treated group as adequate control group and only the female animals for detailed analysis of the combination effect.

\subsubsection{Effect of Single and Combination Treatment on $A \beta$ Deposition}

The different treatments were compared with the isotype control (Figure 1). For both soluble and insoluble brain fractions, a nominal decrease of $A \beta 42$ as well as pGlu3-A $\beta 42$ could be observed for the single treatments. In each case, the decrease was stronger for the combination treatment, and it becomes significant for both $\mathrm{A} \beta$ forms in the soluble fraction $(p \leq 0.01)$ and for pGlu3-A $\beta 42$ also in the insoluble fraction $(p=0.02)$. 


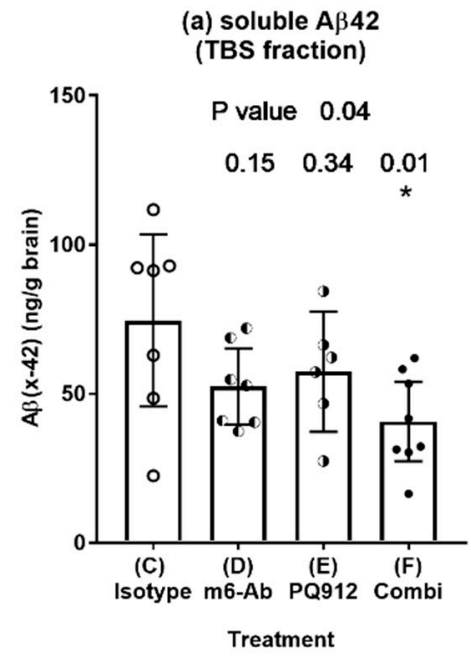

(c) soluble pGlu3-A $\beta$ (TBS fraction)

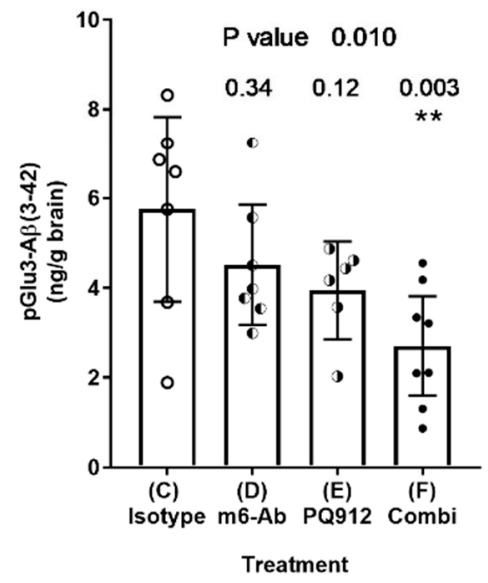

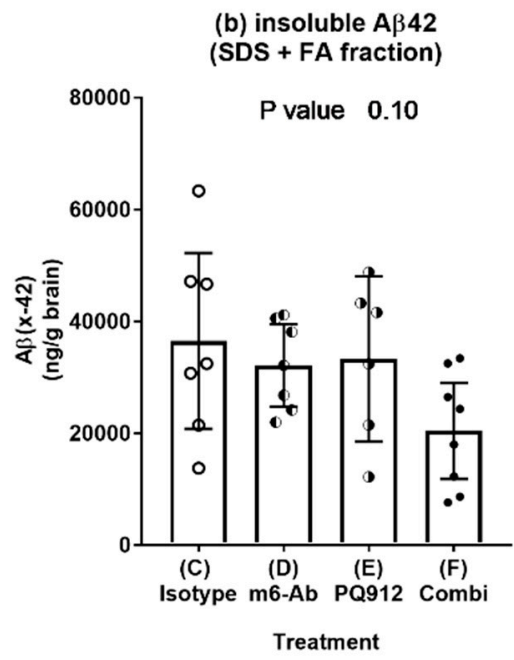

(d) insoluble pGlu3-A $\beta(3-42)$ (SDS + FA fraction)

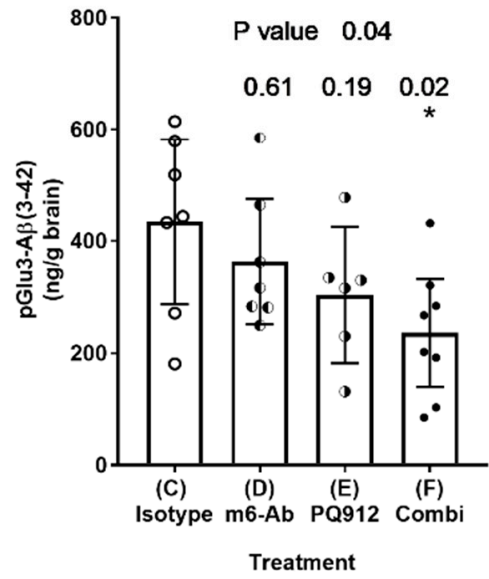

Figure 1. A $\beta$ levels (ELISA) in brain homogenates of $h A P P s l \times h Q C$ mice after treatment with PQ912 (Varoglutamstat), a monoclonal pGlu-A $\beta$ antibody (m6-Ab) or in combination for 16 weeks. Concentration of $A \beta(x-42)(\mathbf{a}, \mathbf{b})$ and pGlu3-A $\beta(3-42)(\mathbf{c}, \mathbf{d})$ in soluble $(\mathbf{a}, \mathbf{c})$ and insoluble $(\mathbf{b}, \mathbf{d})$ brain fractions of 12 -months-old hAPPsl $\times$ hQC mice after 16 weeks of treatment. Dots represent individual levels. Bars and whiskers represent mean $\pm 95 \%$ confidence interval (CI). ANOVA $p$-values are given on top of the graphs. Numbers above the bars represent adjusted $p$-values of Dunnett's post-hoc comparison with the isotype control $\left({ }^{*}-p<0.05,{ }^{* *}-p<0.01\right)$. There is a nominal decrease of total $\mathrm{A} \beta 42$ and pGlu3-A $\beta 42$ by the single treatments. The effect of the combination is stronger compared to the single treatment and becomes significant for $p G l u 3-A \beta$ in both fractions and for $A \beta 42$ in the TBS fraction.

The results of the biochemical analyses are corroborated by the results of the $A \beta$ histochemistry which are shown in Figure 2. Slices of right brain hemispheres of the female animals were stained for $p G l u 3-A \beta(K 17)$ and $A \beta(1-x)(82 E 1)$ (see Figure S4 for representative images). The $\mathrm{A} \beta$ stainings verify the significant increase of pathology over the 16-week treatment period ( $p$-values $<0.01$, $t$-test baseline vs. isotype control, Figure 2 ). In general, lower mean $A \beta$ staining was observed for the treatment with the single agents (PQ912, m6) or the combination. The strongest decrease was observed for the pGlu3-A $\beta$ staining with a significant effect for the combination treatment $(p=0.025)$. 
(a) pGlu3-A $\beta$

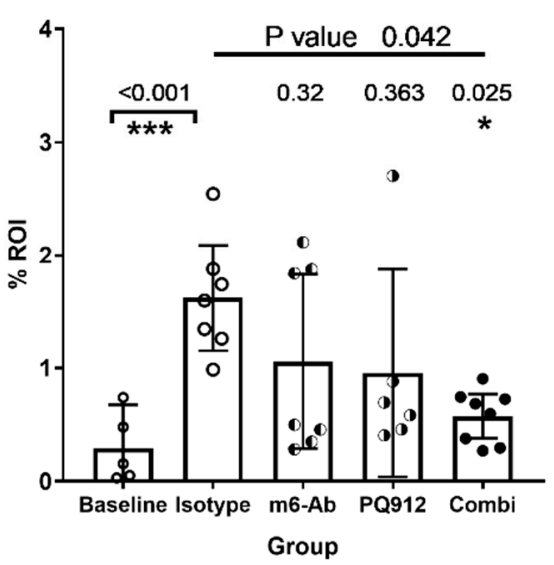

(b) $A \beta(1-x)$

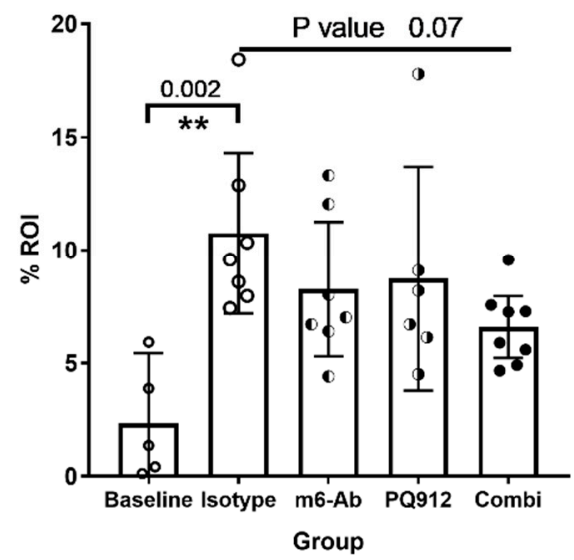

Figure 2. Quantitative immunohistochemistry of brains from $\mathrm{hAPPs} \times \mathrm{hQC}$ mice after treatment with PQ912 (Varoglutamstat), a monoclonal pGlu-A $\beta$ antibody (m6-Ab) or in combination for 16 weeks. (a) K17 stains pGlu3-A $\beta$, (b) 82E1 stains the A $\beta$ N-terminus starting with amino acid 1 [A $\beta(1-x)$ ]. Significant differences between isotype control and baseline ( $t$-test) indicate a development of $A \beta$ pathology over the treatment period. In comparison to the isotype control, nominal lower mean $\mathrm{A} \beta$ staining was found in case of all treatments. The combination treatment resulted in significantly reduced staining for pGlu3-A $\beta$. Kruskal-Wallis test for group comparison (top line $p$-value) with Dunn's test for post-hoc comparison with isotype control ( $p$-values above bars) $\left({ }^{*}-p<0.05\right.$, $* *-p<0.01, * * *-p<0.001)$.

\subsection{Evaluation of Combination Effect According to Bliss}

The Bliss combination indices $\left(\mathrm{CI}_{\text {Bliss }}\right)$ were calculated as described above for $\mathrm{A} \beta$ fractions where a robust reduction (significant ANOVA) of A $\beta$ was observed. Table 1 shows the effects (\% response in comparison to the isotype control) of the single and the combination treatments and the response expected under the Bliss independent conditions (Bliss additivity) as well as the combination index which is calculated by dividing the expected (Bliss additivity) by the observed effect of the combination. Under the assumption that a complete inhibition of pGlu-A $\beta$ formation is possible, combination indices between 0.87 and 0.99 were calculated. The indices support an additive $\left(\mathrm{CI}_{\text {Bliss }}=1\right)$ to slightly synergistic effect of the combination compared with the single treatments.

Table 1. Quantitative evaluation of the combination treatment with PQ912 and m6-Ab for 16 weeks, applying the Bliss model. The parameters are: \% mean response $\left(E_{x}=\%\right.$ decrease of $A \beta$ in respective group $x$ compared to isotype control) for different groups, and calculated Bliss additivity and Bliss combination index $\left(\mathrm{CI}_{\text {Bliss }}\right)$ for $\mathrm{A} \beta 42$ and pGlu-A $\beta 42$ in soluble and insoluble brain fractions; $\mathrm{CI}_{\mathrm{Bliss}}$ are given, if a robust (significant $\mathrm{ANOVA}$ ) effect on $\mathrm{A} \beta$ level was observed (compare to Figures 1 and 2).

\begin{tabular}{|c|c|c|c|c|c|c|}
\hline & $\begin{array}{c}\text { m6-Ab } \\
\left(E_{D}\right)\end{array}$ & $\begin{array}{c}\text { PQ912 } \\
\left(E_{E}\right)\end{array}$ & $\begin{array}{c}\text { Combi } \\
\left(E_{F}\right)\end{array}$ & $\begin{array}{c}\text { Bliss Additivity } \\
\left(\mathrm{E}_{\mathrm{D}}+\mathrm{E}_{\mathrm{E}}-\mathrm{E}_{\mathrm{D}} * \mathrm{E}_{\mathrm{E}}\right)\end{array}$ & $\begin{array}{c}\mathrm{CI}_{\text {Bliss }} \\
\left(\mathrm{E}_{\mathbf{D}}+\mathrm{E}_{\mathrm{E}}-\mathrm{E}_{\mathbf{D}} * \mathrm{E}_{\mathrm{E}}\right) / \mathrm{E}_{\mathbf{F}}\end{array}$ & $\begin{array}{c}\text { Rating of CI According } \\
\text { to Chou [37] }\end{array}$ \\
\hline \multicolumn{7}{|c|}{$\mathrm{A} \beta$ biochemistry } \\
\hline soluble $\mathrm{pE}-\mathrm{A} \beta 42$ & 21.6 & 31.5 & 53.0 & 46.3 & 0.87 & slight synergism \\
\hline insoluble $\mathrm{pE}-\mathrm{A} \beta 42$ & 16.4 & 30.2 & 45.7 & 41.6 & 0.91 & nearly additive \\
\hline soluble $A \beta 42$ & 29.8 & 23.1 & 45.4 & 46.0 & 0.99 & (nearly) additive \\
\hline \multicolumn{7}{|c|}{$\mathrm{A} \beta$ Histochemistry } \\
\hline $\mathrm{pE} 3 \mathrm{~A} \beta(\mathrm{K} 17)$ & 34.6 & 41.0 & 64.5 & 61.4 & 0.95 & nearly additive \\
\hline
\end{tabular}




\section{Discussion}

Compelling evidence suggests that passive immunotherapy represents a promising approach to treat Alzheimer's disease [4,6,38-41]. Among the antibodies which are in advanced clinical development or have recently been approved within the US, it is interesting to note that all of them are specific for aggregated and/or modified forms of $A \beta$. Donanemab is the first antibody, which binds to a highly toxic post-translationally modified $A \beta$, pGlu3-A $\beta$ [28]. The peptide has been shown in numerous studies to significantly accumulate in Alzheimer brain [42-44]. The N-terminal truncation and modification renders the peptide more hydrophobic and prone to aggregation [11,19]. Importantly, some reports imply that the strong formation of pGlu-A $\beta$ appears at stages of pathology closely before manifestation of clinical symptoms, and accumulation correlates with disease better than the "plain" $A \beta$ plaque pathology or accumulation of full-length $A \beta[12,22]$. These findings initiated the development of strategies targeting pGlu-A $\beta$ by small molecule $Q C$ inhibitors or specific antibodies $[24,25]$. Both treatments have been assessed intensively in mouse models, providing evidence for a reduction of $\mathrm{pGlu}-\mathrm{A} \beta$ and concomitant functional improvement in spatial learning $[24,27,35]$.

Although the development of combination therapies for Alzheimer's disease is highly recommended, $[45,46]$ until now only combinations of approved symptomatic therapies with memantine and acetylcholinesterase inhibitors have been investigated in detail [46-50]. Investigations of combinations with disease-modifying drugs are rare. Similar to our approach, Strömberg et al. [51] tested a combination of two A $\beta$ targeting approaches, a BACE inhibitor and a $\gamma$-secretase modulator, and found an additive effect on reduction of $A \beta$ formation in an acute mouse model. However, several aspects support a combination treatment in AD. One prominent supporting feature might be drug safety. For example, with an additive effect of the combination, half dose of each component would achieve a similar degree of therapeutic effect but would keep the individual components more certainly within their respective safety dose range ("therapeutic window"). Especially with regard to ARIA-E (amyloid-related imaging abnormalities—edema) observed with monoclonal antibodies, this feature might be important. Second, tackling pathological A $\beta$ variants or forms by a double-pronged approach could enhance (maximally) the achievable effect by different means. First, interfering very proximal of the pathological pathway by inhibiting production of $\mathrm{pGlu}-\mathrm{A} \beta$ and second, in combination with a more distal effect reducing already existing $\mathrm{pGlu}-\mathrm{A} \beta$ oligomers or -plaques. This could more markedly slow the progression of the disease over a broader dynamic progression range.

There are different methods to assess the effects of drug combinations and interpretation of results depend on the respective definition of additivity. Foucquier and Guedj [52] provided a concise summary of the respective methodological landscape. Beside doseeffect-based strategies based on Loewe additivity [37,53-55] which need an accurate estimation of dose-effect curves and therefore substantial demand on experimental conditions and number of animals, some simpler effect-based approaches are described (highest single agent, combination sub-thresholding, response additivity, Bliss independence) [52]. Among these, we chose the Bliss independence model [56], because for the tested therapies we assume two independent pathways with sigmoidal dose-responses and the same maximum effect $(100 \%$ removal of $A \beta)$. Therefore, we designed an experiment with doses for the single agents which should result in an approximately $30 \%$ pGlu3-A $\beta$ reduction. In the case of Bliss additivity $\left(\mathrm{CI}_{\text {Bliss }}=1\right)$, the combination of the same doses of both agents should result in an $\approx 51 \%$ reduction of pGlu-A $\beta$ (see Equation (1)) whereas lower or higher values for the $\mathrm{CI}_{\text {Bliss }}$ are indicative for synergism or antagonism, respectively.

We used this model here to evaluate a combination study of an antibody targeting pGlu3-A $\beta$, m6 (murine PBD-C06), and Varoglutamstat (PQ912), the first in class glutaminyl cyclase inhibitor in clinical development for treatment of AD [32]. Thereby, the aims of the study are two-fold: (a) To test the hypothesis that pGlu-A $\beta$ antibodies and PQ912 address independent pathways in reducing pGlu3-A $\beta$ pathology and (b) if so, to show that the combination of low, borderline effective doses of the single agents PQ912 or m6 
antibody exert a significant effect on pGlu- $A \beta$ in combination. To address these questions, we used a transgenic model with enhanced $\mathrm{pGlu} 3-\mathrm{A} \beta$ formation, the $\mathrm{hAPPsl} \times \mathrm{hQC}$ double transgenic mouse [27]. The treatment started at an age of 9 months, where the mice already showed amyloid pathology but still accumulate $\mathrm{A} \beta$ over the 16-week treatment period, thus mirroring progress of $\mathrm{A} \beta$ pathology similar to very early stages (preclinical) of human $\mathrm{AD}$. For either of the single treatments at the applied doses, the analyses of the brain tissue by ELISA and histology consistently revealed an insignificant decrease of pGlu3-A $\beta$ and total $A \beta$, whereas the combination of both agents at the same doses generally shows a stronger response than the best single agent treatment. The response exerted by the combination treatment becomes significant in the majority of analyses. According to Foucquier and Guedj [52] these results fulfill the requirements for additivity for both the "highest single agent" as well as the "combination sub-thresholding" approach. The assessment of the Bliss combination index revealed values of nearly 1 , which are indicative for an additive effect of the test compounds addressing two independent pathways both targeting pGlu$\mathrm{A} \beta$ pathology (Table 1). Furthermore, the use of two independent techniques (ELISA, histochemistry) for evaluation of the efficacy of the treatments on reduction of $A \beta$ pathology enables a robust conclusion.

Due to the limited number of animals enrolled in the treatment, the study has some limitations which may be addressed in future studies. The number of dose levels for the single and combination treatments should be increased to identify the most effective dose combination. This also allows the use of a dose-effect based strategy according to Chou [37] for evaluation and could directly demonstrate that a combination with half of the doses of the single treatments will result in same effect as the single treatments. Furthermore, it should be demonstrated also for the combination that the reduction of $A \beta$ leads to an adequate improvement of memory function.

Nevertheless, the data strongly support an additive effect of anti-pGlu-A $\beta$ antibody treatment and glutaminyl cyclase inhibition, i.e., both molecular treatment paradigms basically address different and independent mechanisms to suppress pathology-related pGlu-A $\beta$ and general $A \beta$ accumulation. This conclusion can be explained by the processes, in which both compounds act: $Q C$ inhibition interferes with de novo synthesis of pGlu$A \beta$, the antibody requires the prior formation of the molecule for binding and clearance by microglia or eventually invading monocytes (Figure 3). Although this conclusion appears straightforward, it should be considered that $\mathrm{QC}$ inhibition has been shown to also exert anti-inflammatory effects, mainly driven by suppression of CCL2, resulting in inhibitory effects on monocyte migration and activation $[57,58]$. Although this will be covered in the upcoming studies, the concomitant suppression of inflammation by QC inhibitors without negatively effecting amyloid clearance by phagocytosis might be also favorable for combination with passive immunotherapy to potentially avoid adverse effects such as amyloid-related imaging abnormalities (ARIA) as seen in most clinical trials with plaque-binding anti-amyloid antibodies.

Considering that a part of $\mathrm{pGlu}-\mathrm{A} \beta$ is formed during the secretion process, the combination therapy of these two paradigms might prove favorable to efficiently suppress the accumulation of this toxic species, since it is anticipated that the antibodies are ineffective in clearing intracellular amyloid peptides, as anticipated for tau immunotherapy [32]. The small molecule inhibitor has been shown to penetrate cellular barriers efficiently. Hence, we propose two potential outcomes of a combination of Varoglutamstat with monoclonal antibodies: (i) Reduction of the dose of each compound while maintaining the therapeutic effect by combination treatment as a matter of additivity; and, (ii) possibly, use of the small molecule $\mathrm{QC}$ inhibitor after antibody or combination treatment for long-term prevention of pGlu3-A $\beta$ toxicity ("treat and maintain"). Although requiring further analysis and additional validation in clinics, we here provide a strong rationale for the evaluation of additional drug combinations for treatment of AD. 


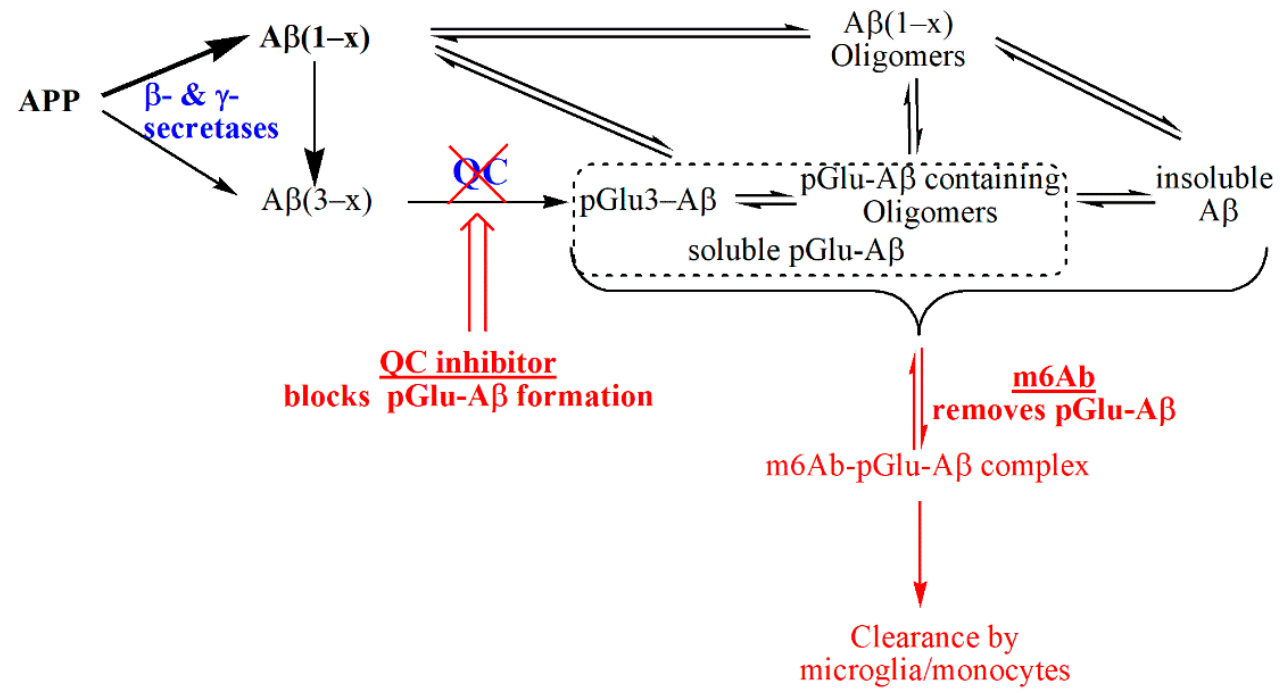

Figure 3. Schematic representation of the molecular pathways for reduction of pGlu3-A $\beta$ by QC inhibition and anti-pGlu3-immunotherapy (m6Ab). The current study supports the concept that both strategies act independently and are additive. The formation of pGlu-A $\beta$ occurs after APP cleavage by sheddases such as BACE1 or meprin $\beta$, which may lead directly to $N$-truncated $A \beta$ forms and the pGlu3-A $\beta$ precursor, $A \beta(3-x)$. While $Q C$ inhibition prevents de novo-synthesis of $p G l u 3-A \beta$, which reduces formation of toxic oligomers and coaggregation with other $A \beta$ forms, the $p G l u 3-A \beta$ specific antibody prevents aggregation and elicits clearance of extracellular, soluble pGlu3-A $\beta$ containing aggregates by opsonization and phagocytosis by microglia and/or monocytes.

\section{Materials and Methods}

\subsection{Materials}

The glutaminyl cyclase inhibitor PQ912 [(S)-1-(1H-benzo[d]imidazol-5-yl)5(4propoxyphenyl)imidazolidin-2-one] was used in this study. PQ912 was produced by Carbogen Amcis (Aarau, Switzerland). Chow containing $0.8 \mathrm{~g}$ PQ912 per kg was prepared by Ssniff Spezialdiäten (Soest, Germany) on base of the Ssniff R/M-H (V1534) diet.

The mouse IgG2a variant of the murine anti-pGlu3A $\beta$ antibody PBD-C06 (PBD-C06.02; m6) has been produced as described previously [34,59].

\subsection{Animal Experiments and Tissue Collection}

hAPPsl $\times$ hQC mice were used in this study [27]. These mice express human amyloid precursor protein (hAPP)695 bearing the Swedish and London (sl) mutation and human $\mathrm{QC}$, both under control of the Thy-1 promoter. The mice develop pGlu-A $\beta$ positive deposits starting at an age of 6 to 7 months, the percentage of pGlu3-A $\beta$ ranges, depending on the age of the animals, from $0.1-2 \%$ (https://qpsneuro.com/in-vivo-services/animalmodels / app-transgenic-mouse-models / appsl- $x$-hqc-transgenic-mouse-model/, access on 28 October 2021). Animals were housed at QPS Austria (Grambach) in individually ventilated cages on standardized rodent bedding supplied by J. Rettenmaier \& Söhne (Rosenberg, Germany) (21 ${ }^{\circ} \mathrm{C}, 40-70 \%$ rel. humidity, lights on from 6 a.m. to 6 p.m.). Pelleted standard rodent chow or compound containing chow (Ssniff, Soest, Germany) as well as normal tap water was available ad libitum.

In an initial dose-finding study, eight $\mathrm{hAPPsl} \times \mathrm{hQC}$ mice per group (male and female) were used. Animals were treated for 16 weeks starting at an age of $9 \pm 1$ months. The three groups were either treated with vehicle, $150 \mu \mathrm{g}$ anti-pGlu3-A $\beta$ antibody (m6), or $500 \mu \mathrm{g}$ m6 per animal once a week by i.p. injection (application volume 200-250 $\mu \mathrm{L}$ ). At the end of the study, animals were sacrificed, brains collected and subjected to A $\beta$ extraction followed by ELISA analysis as described below [27].

In a combination study investigating the efficacy of QC inhibitor (PQ912) and antipGlu3-A $\beta$ antibody (m6), 70 hAPPsl $\times$ hQC mice of both sexes aged between 8 and 9 months 
were allocated to five treatment groups (Table 2). Either IgG2a isotype control (C, E) or m6 (D, F) or vehicle (B) was administered once a week i.p. for 16 weeks. In addition to the i.p. treatment, two groups of animals (E and F) received PQ912 orally via food pellets for the 16-week treatment period. Body weight and food consumption was assessed once a week. Another subset of 10 naive animals was subjected to tissue sampling at the time point of treatment start (Baseline group A). Because of premature death, one additional animal was included in group $\mathrm{F}$ to compensate for the lost animal.

Table 2. Overview on treatment groups in the combination experiment.

\begin{tabular}{ccccc}
\hline Group & $\mathbf{N}$ & Chow & Treatment & Time of Tissue \\
Sampling
\end{tabular}

At the end of the 16-week treatment period, animals were sacrificed and plasma and brain tissue collected and analyzed as described previously [27].

\subsection{A $\beta$ Extraction and ELISAs}

Briefly, for $\mathrm{A} \beta$ extraction, one brain hemisphere without cerebellum was homogenized in TBS (20 mM Tris, $137 \mathrm{mM} \mathrm{NaCl}$, pH 7.6; $2.5 \mathrm{~mL}$, Dounce homogenizer) containing protease inhibitor cocktail (Complete Mini, Roche) and 0.1 mM AEBSF, sonicated and centrifuged at $75,500 \times g$ for $1 \mathrm{~h}$ at $4{ }^{\circ} \mathrm{C}$. The supernatant (TBS soluble $\mathrm{A} \beta$ fraction) was stored at $-80^{\circ} \mathrm{C}$. Insoluble $\mathrm{A} \beta$ species were sequentially extracted from the pellet using $2.5 \mathrm{~mL} 2 \% \mathrm{SDS}$ in distilled water (SDS fraction), and $0.5 \mathrm{~mL} \mathrm{70 \%} \mathrm{formic} \mathrm{acid} \mathrm{(FA} \mathrm{fraction).}$ The formic acid extract was neutralized by addition of $3.5 \mathrm{M}$ Tris solution and diluted to a final volume of $12.95 \mathrm{~mL}$ using ELISA blocking buffer (Pierce, Cat.No. 37571, ThermoFisher, Dreieich, Germany). A $\beta(x-42)$ and pGlu3-A $\beta(3-42)$ were determined in all brain fractions by specific sandwich ELISAs (IBL, Hamburg, Germany) according to the manufacturer's manual. Individual brain content of $A \beta$ (ng/g) was calculated by considering the brain wet weight of each sample. The sum of A $\beta$ detected in the SDS and FA fractions were considered as the insoluble pool of $\mathrm{A} \beta$.

\subsection{Immunohistochemistry}

Immunohistochemistry using the antibodies K17 (pGlu3-A $\beta$ specific, IgG2b, Vivoryon N.V., Germany) and 82E1 (A $\beta(1-\mathrm{x})$ specific; IBL Hamburg, Germany) was performed as described previously [35].

Briefly, $10-\mu \mathrm{m}$-thick brain sagittal cryosections were cut using a Leica CM1850 cryostat and mounted on Colorfrost Plus slides (Fisher Scientific). Sections were air-dried for $20 \mathrm{~min}$ and washed with TBS for $2 \times 5 \mathrm{~min}$. Immunohistochemistry was carried out using the Elite ABC Kit (Vector Laboratories, Burlingame, CA, USA). Quantitative image analysis of the percent area of immunoreactivity (IR) was obtained by using the Bioquant image analysis software (Nashville, TN, USA) on six sections per mouse at three equidistant planes for hippocampus and frontal cortex.

\subsection{Data Handling, Statistics, and Calculations}

The data obtained from the initial dose finding study were analyzed using ANOVA and Dunnett's post-hoc comparison of m6-treated groups with the vehicle-treated group.

For statistical data evaluation of efficacy in the combination study, a stepwise approach was used. First, a 2-way-ANOVA with the baseline, the PBS-treated, and the isotypeantibody-treated group using time and gender as factors was done to evaluate whether 
the animals develop $\mathrm{A} \beta$ pathology over duration of treatment and if there were gender differences or differences between PBS and isotype treatment. This analysis was performed for the insoluble $A \beta$ fractions. A significant $(\alpha=0.05)$ increase of pathology after 16 weeks compared to baseline, as observed for the female mice, was considered as prerequisite for further analysis. In the second step the groups treated with a single agent (m6 or PQ912) and the combination treatment were compared with the isotype antibody treated control using one-way ANOVA and Dunnett's post-hoc comparison of treatment groups with the isotype control group (respective non-parametric tests for histochemical analyses). Very low total $A \beta 42$ levels were detected at end of treatment in three 12 months old animals (one male and one female, group $\mathrm{E}$ and one female, group $\mathrm{D}$ ). In these mice the total $\mathrm{A} \beta 42$ levels were lower than half of the lowest value in the baseline group $(433 \mathrm{ng} / \mathrm{g})$. It is assumed that these animals did not express the APP transgene sufficiently. Therefore, these animals were excluded from further analyses.

For statistical evaluations and preparation of graphs Prism 7 for Windows (V7.03, GraphPad Software Inc., San Diego, CA, USA) was used.

\section{Evaluation of Additivity}

Additivity of treatments was evaluated according to the Bliss model which assumes that both treatments independently contribute to a common result [52]. The Bliss combination index $\left(\mathrm{CI}_{\text {Bliss }}\right)$ was calculated according to the following formula:

$$
\mathrm{CI}_{\mathrm{Bliss}}=\left(\mathrm{E}_{\mathrm{A}}+\mathrm{E}_{\mathrm{B}}-\mathrm{E}_{\mathrm{A}} * \mathrm{E}_{\mathrm{B}}\right) / \mathrm{E}_{\mathrm{AB}}
$$

were $E_{A}, E_{B}$, and $E_{A B}$ are the effects of treatment $A$, treatment $B$, and the combination treatment $A B$, respectively. Using this equation, a combination index of 1 indicates additivity of the single treatments in the combination, a $\mathrm{CI}_{\text {Bliss }}<1$ indicates synergism and an index $>1$ antagonism. The effect was calculated as \% decrease in A $\beta$ compared to the isotype control:

$$
\mathrm{E}(\%)=100 \times\left(1-\mathrm{A} \beta_{\text {Treatment }} / \mathrm{A} \beta_{\text {Isotype control }}\right)
$$

Supplementary Materials: The following are available online at https:/ / www.mdpi.com/article/10 .3390/ijms222111791/s1.

Author Contributions: Conceptualization, S.S., I.L., C.A.L., T.H.; methodology, T.H., J.-U.R., M.S., F.P., B.H.-P., C.A.L., S.S.; formal analysis, T.H., S.S., C.A.L.; investigation, F.P., K.M., B.H.-P., C.A.L., S.S.; resources, J.-U.R., M.S., B.H.-P., I.L., C.A.L., S.S.; writing—original draft preparation, T.H., S.S.; writing-review and editing; T.H., S.S., C.A.L., I.L.; supervision, I.L., S.S., T.H., C.A.L., B.H.-P. All authors have read and agreed to the published version of the manuscript.

Funding: The work of C.A.L. was funded by the NIH/NIA, grant numbers: NIH/NIA R01 AG040092 (CAL) and NIH/NIA RF1 AG058657 (CAL).

Institutional Review Board Statement: The QPS Austria facility in Grambach is fully accredited by the Association for Assessment and Accreditation of Laboratory Animal Care (AAALAC). All procedures in this study complied with the Animal Care and Welfare Committee. Animals were maintained according to the animal welfare regulations of the Ministry of Science of the Austrian government (TVG 2012 in the appropriate valid version).

Informed Consent Statement: Not applicable.

Data Availability Statement: Data of the study are available from the corresponding authors upon reasonable request.

Acknowledgments: Antje Meyer, Martin Kleinschmidt and Swiss Bioquant (Reinach, Switzerland) for managing and measurement of compound and antibody analytics, Anke Piechotta for generating the $\mathrm{m} 6$ cell line. Maren Schroeder for assistance in preparing brain slices and images.

Conflicts of Interest: T.H. is employee of Vivoryon N.V., S.S. and J.R. serves as consultants to Vivoryon N.V., I.L. holds stock of Vivoryon N.V., C.A.L. receives antibodies for her NIH-funded 
research from Vivoryon Therapeutics as a gift-in-kind and received unrestricted funding from Vivoryon Therapeutics (formerly Probiodrug AG) for her research contribution to this study. All other authors declare no conflict of interest.

\section{References}

1. GBD 2016 Dementia Collaborators. Global, regional, and national burden of Alzheimer's disease and other dementias, 1990-2016: A systematic analysis for the Global Burden of Disease Study 2016. Lancet. Neurol. 2019, 18, 88-106. [CrossRef]

2. Sevigny, J.; Chiao, P.; Bussiere, T.; Weinreb, P.H.; Williams, L.; Maier, M.; Dunstan, R.; Salloway, S.; Chen, T.; Ling, Y.; et al. The antibody aducanumab reduces Abeta plaques in Alzheimer's disease. Nature 2016, 537, 50-56. [CrossRef] [PubMed]

3. Budd, H.S.; O'Gorman, J.; Chiao, P.; Bussiere, T.; von Rosenstiel, P.; Tian, Y.; Zhu, Y.; von Hehn, C.; Gheuens, S.; Skordos, L.; et al. Clinical Development of Aducanumab, an Anti-Abeta Human Monoclonal Antibody Being Investigated for the Treatment of Early Alzheimer's Disease. J. Prev. Alzheimer's Dis. 2017, 4, 255-263.

4. Cummings, J.; Aisen, P.; Lemere, C.; Atri, A.; Sabbagh, M.; Salloway, S. Aducanumab produced a clinically meaningful benefit in association with amyloid lowering. Alzheimer's Res. Ther. 2021, 13, 98. [CrossRef] [PubMed]

5. Selkoe, D.J.; Hardy, J. The amyloid hypothesis of Alzheimer's disease at 25 years. EMBO Mol. Med. 2016, 8, 595-608. [CrossRef]

6. Mintun, M.A.; Lo, A.C.; Duggan Evans, C.; Wessels, A.M.; Ardayfio, P.A.; Andersen, S.W.; Shcherbinin, S.; Sparks, J.; Sims, J.R.; Brys, M.; et al. Donanemab in Early Alzheimer's Disease. N. Engl. J. Med. 2021, 384, 1691-1704. [CrossRef] [PubMed]

7. Lowe, S.L.; Willis, B.A.; Hawdon, A.; Natanegara, F.; Chua, L.; Foster, J.; Shcherbinin, S.; Ardayfio, P.; Sims, J.R. Donanemab (LY3002813) dose-escalation study in Alzheimer's disease. Alzheimer's Dement. Transl. Res. Clin. Interv. 2021, 7, e12112. [CrossRef]

8. Nussbaum, J.M.; Schilling, S.; Cynis, H.; Silva, A.; Swanson, E.; Wangsanut, T.; Tayler, K.; Wiltgen, B.; Hatami, A.; Ronicke, R.; et al. Prion-like behaviour and tau-dependent cytotoxicity of pyroglutamylated amyloid-beta. Nature 2012, 485, 651-655. [CrossRef] [PubMed]

9. Wittnam, J.L.; Portelius, E.; Zetterberg, H.; Gustavsson, M.K.; Schilling, S.; Koch, B.; Demuth, H.U.; Blennow, K.; Wirths, O.; Bayer, T.A. Pyroglutamate amyloid beta (Abeta) aggravates behavioral deficits in transgenic amyloid mouse model for Alzheimer disease. J. Biol. Chem. 2012, 287, 8154-8162. [CrossRef]

10. Becker, A.; Kohlmann, S.; Alexandru, A.; Jagla, W.; Canneva, F.; Bauscher, C.; Cynis, H.; Sedlmeier, R.; Graubner, S.; Schilling, S.; et al. Glutaminyl cyclase-mediated toxicity of pyroglutamate-beta amyloid induces striatal neurodegeneration. BMC Neurosci. 2013, 14, 108. [CrossRef]

11. Schlenzig, D.; Manhart, S.; Cinar, Y.; Kleinschmidt, M.; Hause, G.; Willbold, D.; Funke, S.A.; Schilling, S.; Demuth, H.U. Pyroglutamate formation influences solubility and amyloidogenicity of amyloid peptides. Biochemistry 2009, 48, 7072-7078. [CrossRef] [PubMed]

12. Morawski, M.; Schilling, S.; Kreuzberger, M.; Waniek, A.; Jager, C.; Koch, B.; Cynis, H.; Kehlen, A.; Arendt, T.; Hartlage-Rubsamen, M.; et al. Glutaminyl cyclase in human cortex: Correlation with (pGlu)-amyloid-beta load and cognitive decline in Alzheimer's disease. J. Alzheimer's Dis. 2014, 39, 385-400. [CrossRef]

13. Morawski, M.; Hartlage-Rubsamen, M.; Jager, C.; Waniek, A.; Schilling, S.; Schwab, C.; McGeer, P.L.; Arendt, T.; Demuth, H.U.; Rossner, S. Distinct glutaminyl cyclase expression in Edinger-Westphal nucleus, locus coeruleus and nucleus basalis Meynert contributes to pGlu-Abeta pathology in Alzheimer's disease. Acta Neuropathol. 2010, 120, 195-207. [CrossRef] [PubMed]

14. Wirths, O.; Breyhan, H.; Cynis, H.; Schilling, S.; Demuth, H.U.; Bayer, T.A. Intraneuronal pyroglutamate-Abeta 3-42 triggers neurodegeneration and lethal neurological deficits in a transgenic mouse model. Acta Neuropathol. 2009, 118, 487-496. [CrossRef]

15. Jawhar, S.; Wirths, O.; Schilling, S.; Graubner, S.; Demuth, H.U.; Bayer, T.A. Overexpression of glutaminyl cyclase, the enzyme responsible for pyroglutamate $\mathrm{A}\{$ beta\} formation, induces behavioral deficits, and glutaminyl cyclase knock-out rescues the behavioral phenotype in 5XFAD mice. J. Biol. Chem. 2011, 286, 4454-4460. [CrossRef] [PubMed]

16. Valverde, A.; Dunys, J.; Lorivel, T.; Debayle, D.; Gay, A.S.; Lacas-Gervais, S.; Roques, B.P.; Chami, M.; Checler, F. Aminopeptidase A contributes to biochemical, anatomical and cognitive defects in Alzheimer's disease (AD) mouse model and is increased at early stage in sporadic AD brain. Acta Neuropathol. 2021, 141, 823-839. [CrossRef]

17. Antonyan, A.; Schlenzig, D.; Schilling, S.; Naumann, M.; Sharoyan, S.; Mardanyan, S.; Demuth, H.U. Concerted action of dipeptidyl peptidase IV and glutaminyl cyclase results in formation of pyroglutamate-modified amyloid peptides in vitro. Neurochem. Int. 2018, 113, 112-119. [CrossRef]

18. Schlenzig, D.; Cynis, H.; Hartlage-Rübsamen, M.; Zeitschel, U.; Menge, K.; Fothe, A.; Ramsbeck, D.; Spahn, C.; Wermann, M.; Roßner, S.; et al. Dipeptidyl-Peptidase Activity of Meprin $\beta$ Links N-truncation of A $\beta$ with Glutaminyl Cyclase-Catalyzed pGlu-A $\beta$ Formation. J. Alzheimers's Dis. 2018, 66, 359-375. [CrossRef] [PubMed]

19. Schlenzig, D.; Ronicke, R.; Cynis, H.; Ludwig, H.H.; Scheel, E.; Reymann, K.; Saido, T.; Hause, G.; Schilling, S.; Demuth, H.U. N-Terminal pyroglutamate formation of Abeta38 and Abeta40 enforces oligomer formation and potency to disrupt hippocampal long-term potentiation. J. Neurochem. 2012, 121, 774-784. [CrossRef]

20. Grochowska, K.M.; Yuanxiang, P.; Bär, J.; Raman, R.; Brugal, G.; Sahu, G.; Schweizer, M.; Bikbaev, A.; Schilling, S.; Demuth, H.; et al. Posttranslational modification impact on the mechanism by which amyloid- $\beta$ induces synaptic dysfunction. EMBO Rep. 2017, 18, 962-981. [CrossRef] 
21. Russo, C.; Violani, E.; Salis, S.; Venezia, V.; Dolcini, V.; Damonte, G.; Benatti, U.; Arrigo, C.; Patrone, E.; Carlo, P.; et al. Pyroglutamate-modified amyloid beta-peptides-AbetaN3(pE)-strongly affect cultured neuron and astrocyte survival. J. Neurochem. 2002, 82, 1480-1489. [CrossRef]

22. Rijal, U.A.; Kosterin, I.; Kumar, S.; von Arnim, C.A.; Yamaguchi, H.; Fandrich, M.; Walter, J.; Thal, D.R. Biochemical stages of amyloid-beta peptide aggregation and accumulation in the human brain and their association with symptomatic and pathologically preclinical Alzheimer's disease. Brain 2014, 137, 887-903. [CrossRef]

23. Mandler, M.; Walker, L.; Santic, R.; Hanson, P.; Upadhaya, A.R.; Colloby, S.J.; Morris, C.M.; Thal, D.R.; Thomas, A.J.; Schneeberger, A.; et al. Pyroglutamylated amyloid-beta is associated with hyperphosphorylated tau and severity of Alzheimer's disease. Acta Neuropathol. 2014, 128, 67-79. [CrossRef]

24. Schilling, S.; Zeitschel, U.; Hoffmann, T.; Heiser, U.; Francke, M.; Kehlen, A.; Holzer, M.; Hutter-Paier, B.; Prokesch, M.; Windisch, M.; et al. Glutaminyl cyclase inhibition attenuates pyroglutamate Abeta and Alzheimer's disease-like pathology. Nat. Med. 2008, 14, 1106-1111. [CrossRef] [PubMed]

25. Frost, J.L.; Liu, B.; Kleinschmidt, M.; Schilling, S.; Demuth, H.U.; Lemere, C.A. Passive immunization against pyroglutamate-3 amyloid-beta reduces plaque burden in Alzheimer-like transgenic mice: A pilot study. Neurodegener. Dis. 2012, 10, 265-270. [CrossRef] [PubMed]

26. Frost, J.L.; Liu, B.; Rahfeld, J.U.; Kleinschmidt, M.; O’Nuallain, B.; Le, K.X.; Lues, I.; Caldarone, B.J.; Schilling, S.; Demuth, H.U.; et al. An anti-pyroglutamate-3 Abeta vaccine reduces plaques and improves cognition in APPswe/PS1DeltaE9 mice. Neurobiol. Aging 2015, 36, 3187-3199. [CrossRef] [PubMed]

27. Hoffmann, T.; Meyer, A.; Heiser, U.; Kurat, S.; Bohme, L.; Kleinschmidt, M.; Buhring, K.U.; Hutter-Paier, B.; Farcher, M.; Demuth, H.U.; et al. Glutaminyl Cyclase Inhibitor PQ912 Improves Cognition in Mouse Models of Alzheimer's Disease-Studies on Relation to Effective Target Occupancy. J. Pharmacol. Exp. Ther. 2017, 362, 119-130. [CrossRef]

28. Demattos, R.B.; Lu, J.; Tang, Y.; Racke, M.M.; Delong, C.A.; Tzaferis, J.A.; Hole, J.T.; Forster, B.M.; McDonnell, P.C.; Liu, F.; et al. A Plaque-Specific Antibody Clears Existing beta-amyloid Plaques in Alzheimer's Disease Mice. Neuron 2012, 76, 908-920. [CrossRef] [PubMed]

29. Pohl, T.; Zimmer, M.; Mugele, K.; Spiess, J. Primary structure and functional expression of a glutaminyl cyclase. Proc. Natl. Acad. Sci. USA 1991, 88, 10059-10063. [CrossRef] [PubMed]

30. Bockers, T.M.; Kreutz, M.R.; Pohl, T. Glutaminyl-cyclase expression in the bovine/porcine hypothalamus and pituitary. J. Neuroendocr. 1995, 7, 445-453. [CrossRef] [PubMed]

31. De Kimpe, L.; Bennis, A.; Zwart, R.; van Haastert, E.S.; Hoozemans, J.J.; Scheper, W. Disturbed Ca2+ homeostasis increases glutaminyl cyclase expression; connecting two early pathogenic events in Alzheimer's disease in vitro. PLoS ONE 2012, 7, e44674. [CrossRef]

32. Lues, I.; Weber, F.; Meyer, A.; Bühring, U.; Hoffmann, T.; Kühn-Wache, K.; Manhart, S.; Heiser, U.; Pokorny, R.; Chiesa, J.; et al. A phase 1 study to evaluate the safety and pharmacokinetics of PQ912, a glutaminyl cyclase inhibitor, in healthy subjects. Alzheimer's Dement. Transl. Res. Clin. Interv. 2015, 1, 182-195. [CrossRef]

33. Scheltens, P.; Hallikainen, M.; Grimmer, T.; Duning, T.; Gouw, A.A.; Teunissen, C.E.; Wink, A.M.; Maruff, P.; Harrison, J.; Van Baal, C.M.; et al. Safety, tolerability and efficacy of the glutaminyl cyclase inhibitor PQ912 in Alzheimer's disease: Results of a randomized, double-blind, placebo-controlled phase 2a study. Alzheimer's Res. Ther. 2018, 10, 107. [CrossRef] [PubMed]

34. Hettmann, T.; Gillies, S.D.; Kleinschmidt, M.; Piechotta, A.; Makioka, K.; Lemere, C.A.; Schilling, S.; Rahfeld, J.U.; Lues, I. Development of the clinical candidate PBD-C06, a humanized pGlu3-A $\beta$-specific antibody against Alzheimer's disease with reduced complement activation. Sci. Rep. 2020, 10, 3294. [CrossRef] [PubMed]

35. Crehan, H.; Liu, B.; Kleinschmidt, M.; Rahfeld, J.U.; Le, K.X.; Caldarone, B.J.; Frost, J.L.; Hettmann, T.; Hutter-Paier, B.; O’Nuallain, B.; et al. Effector function of anti-pyroglutamate-3 A $\beta$ antibodies affects cognitive benefit, glial activation and amyloid clearance in Alzheimer's-like mice. Alzheimer's Res. Ther. 2020, 12, 12. [CrossRef]

36. Sturchler-Pierrat, C.; Staufenbiel, M. Pathogenic mechanisms of Alzheimer's disease analyzed in the APP23 transgenic mouse model. Ann. N. Y. Acad. Sci. 2000, 920, 134-139. [CrossRef]

37. Chou, T.C. Theoretical basis, experimental design, and computerized simulation of synergism and antagonism in drug combination studies. Pharmacol. Rev. 2006, 58, 621-681. [CrossRef] [PubMed]

38. Swanson, C.J.; Zhang, Y.; Dhadda, S.; Wang, J.; Kaplow, J.; Lai, R.Y.K.; Lannfelt, L.; Bradley, H.; Rabe, M.; Koyama, A.; et al. A randomized, double-blind, phase $2 \mathrm{~b}$ proof-of-concept clinical trial in early Alzheimer's disease with lecanemab, an anti-A $\beta$ protofibril antibody. Alzheimer's Res. Ther. 2021, 13, 80. [CrossRef] [PubMed]

39. Schilling, S.; Rahfeld, J.U.; Lues, I.; Lemere, C.A. Passive A $\beta$ immunotherapy: Current achievements and future perspectives. Molecules 2018, 23, 1068. [CrossRef] [PubMed]

40. Cummings, J.; Lee, G.; Mortsdorf, T.; Ritter, A.; Zhong, K. Alzheimer's disease drug development pipeline: 2017. Alzheimer's Dement. Transl. Res. Clin. Interv. 2017, 3, 367-384. [CrossRef] [PubMed]

41. Tolar, M.; Abushakra, S.; Hey, J.A.; Porsteinsson, A.; Sabbagh, M. Aducanumab, gantenerumab, BAN2401, and ALZ-801-The first wave of amyloid-targeting drugs for Alzheimer's disease with potential for near term approval. Alzheimer's Res. Ther. 2020, 12, 95. [CrossRef] [PubMed] 
42. Piccini, A.; Russo, C.; Gliozzi, A.; Relini, A.; Vitali, A.; Borghi, R.; Giliberto, L.; Armirotti, A.; D’Arrigo, C.; Bachi, A.; et al. \{beta\}-Amyloid Is Different in Normal Aging and in Alzheimer Disease. J. Biol. Chem. 2005, 280, 34186-34192. [CrossRef] [PubMed]

43. Saido, T.C.; Iwatsubo, T.; Mann, D.M.; Shimada, H.; Ihara, Y.; Kawashima, S. Dominant and differential deposition of distinct beta-amyloid peptide species, A beta N3(pE), in senile plaques. Neuron 1995, 14, 457-466. [CrossRef]

44. Guntert, A.; Dobeli, H.; Bohrmann, B. High sensitivity analysis of amyloid-beta peptide composition in amyloid deposits from human and PS2APP mouse brain. Neuroscience 2006, 143, 461-475. [CrossRef]

45. Cummings, J.L.; Tong, G.; Ballard, C. Treatment Combinations for Alzheimer's Disease: Current and Future Pharmacotherapy Options. J. Alzheimer's Dis. 2019, 67, 779-794. [CrossRef]

46. Hendrix, J.A.; Bateman, R.J.; Brashear, H.R.; Duggan, C.; Carrillo, M.C.; Bain, L.J.; DeMattos, R.; Katz, R.G.; Ostrowitzki, S.; Siemers, E.; et al. Challenges, solutions, and recommendations for Alzheimer's disease combination therapy. Alzheimer's Dement. 2016, 12, 623-630. [CrossRef]

47. Chen, R.; Chan, P.T.; Chu, H.; Lin, Y.C.; Chang, P.C.; Chen, C.Y.; Chou, K.R. Treatment effects between monotherapy of donepezil versus combination with memantine for Alzheimer disease: A meta-analysis. PLoS ONE 2017, 12, e0183586. [CrossRef]

48. Atri, A.; Hendrix, S.B.; Pejović, V.; Hofbauer, R.K.; Edwards, J.; Molinuevo, J.L.; Graham, S.M. Cumulative, additive benefits of memantine-donepezil combination over component monotherapies in moderate to severe Alzheimer's dementia: A pooled area under the curve analysis. Alzheimer's Res. Ther. 2015, 7, 1-12. [CrossRef]

49. Shao, Z.-Q. Comparison of the efficacy of four cholinesterase inhibitors in combination with memantine for the treatment of Alzheimer's disease. Int. J. Clin. Exp. Med. 2015, 8, 2944-2948.

50. Riverol, M.; Slachevsky, A.; López, O.L. Efficacy and Tolerability of a Combination Treatment of Memantine and Donepezil for Alzheimer's Disease: A Literature Review Evidence. Eur. Neurol. J. 2014, 23, 15-19.

51. Strömberg, K.; Eketjäll, S.; Georgievska, B.; Tunblad, K.; Eliason, K.; Olsson, F.; Radesäter, A.C.; Klintenberg, R.; Arvidsson, P.I.; Von Berg, S.; et al. Combining an amyloid-beta $(\mathrm{A} \beta)$ cleaving enzyme inhibitor with a $\gamma$-secretase modulator results in an additive reduction of $\mathrm{A} \beta$ production. FEBS J. 2015, 282, 65-73. [CrossRef] [PubMed]

52. Foucquier, J.; Guedj, M. Analysis of drug combinations: Current methodological landscape. Pharmacol. Res. Perspect. 2015, 3, e00149. [CrossRef] [PubMed]

53. Loewe, S.; Muischnek, H. Über Kombinationswirkungen. Naunyn. Schmiedebergs. Arch. Exp. Pathol. Pharmakol. 1926, 114, 313-326. [CrossRef]

54. Chou, T.C.; Talalay, P. Quantitative analysis of dose-effect relationships: The combined effects of multiple drugs or enzyme inhibitors. Adv. Enzyme Regul. 1984, 22, 27-55. [CrossRef]

55. Tallarida, R.J. An overview of drug combination analysis with isobolograms. J. Pharmacol. Exp. Ther. 2006, 319, 1-7. [CrossRef] [PubMed]

56. BLISS, C.I. THE TOXICITY OF POISONS APPLIED JOINTLY. Ann. Appl. Biol. 1939, 26, 585-615. [CrossRef]

57. Cynis, H.; Hoffmann, T.; Friedrich, D.; Kehlen, A.; Gans, K.; Kleinschmidt, M.; Rahfeld, J.U.; Wolf, R.; Wermann, M.; Stephan, A.; et al. The isoenzyme of glutaminyl cyclase is an important regulator of monocyte infiltration under inflammatory conditions. EMBO Mol. Med. 2011, 3, 545-558. [CrossRef] [PubMed]

58. Cynis, H.; Kehlen, A.; Haegele, M.; Hoffmann, T.; Heiser, U.; Fujii, M.; Shibazaki, Y.; Yoneyama, H.; Schilling, S.; Demuth, H.U. Inhibition of Glutaminyl Cyclases alleviates CCL2-mediated inflammation of non-alcoholic fatty liver disease in mice. Int. J. Exp. Pathol. 2013, 94, 217-225. [CrossRef]

59. Piechotta, A.; Parthier, C.; Kleinschmidt, M.; Gnoth, K.; Pillot, T.; Lues, I.; Demuth, H.U.; Schilling, S.; Rahfeld, J.U.; Stubbs, M.T. Structural and functional analyses of pyroglutamate-amyloid- $\beta$-specific antibodies as a basis for Alzheimer immunotherapy. $J$. Biol. Chem. 2017, 292, 12713-12724. [CrossRef] 